\title{
Renal functional reserve in patients with severe chronic obstructive pulmonary disease
}

\author{
R A Sharkey, E M T Mulloy, I A Kilgallen, S J O’Neill
}

\begin{abstract}
Background - Renal functional reserve is the normal increase in renal blood flow after a protein load, and reduced or absent renal functional reserve is an early index of renal impairment. Renal blood flow is frequently reduced during acute oedematous exacerbations of chronic obstructive pulmonary disease (COPD). It is possible that patients with severe COPD in the stable state may have a reduced or absent renal functional reserve which could be a factor in oedema formation. Methods - Sixteen stable patients with severe COPD and five normal controls were studied. The mean (SD) arterial oxygen and carbon dioxide tensions $\left(\mathrm{PaO}_{2}, \mathrm{PaCO}_{2}\right)$ and forced expiratory volume in one second $\left(F_{E V}\right)$ of patients with COPD were 8.1 (1.04) kPa, $6.3(0.69) \mathrm{kPa}$, and $0.74(0.27) 1$, respectively. The pulsatility index (PI), an index of renovascular resistance, was measured non-invasively by Doppler ultrasonography at baseline and at intervals after a protein load of $250 \mathrm{~g}$ steak.

Results - The PI fell after the protein load in the normal subjects from $1.04(0.19)$ to $0.84(0.17)$, mean difference $0.20,95 \%$ confidence interval of difference (CI) 0.14 to $0.27, \mathrm{p}<0.001$. In the COPD group there was no change; baseline $P I=1.04(0.16)$, PI after protein load $=1.08(0.19)$, mean difference $=-0.04,95 \% \mathrm{CI}-0.11$ to 0.04 , $\mathbf{p}=$ NS. Six of the patients with COPD were normocapnic and 10 were hypercapnic $\left(\mathrm{PaCO}_{2} \geq 6.0 \mathrm{kPa}\right)$. The normocapnic patients had no significant change in PI (baseline $P I=1.07(0.15), P I$ after protein load $=1.01(0.16)$, mean difference $=0.06$, $95 \% \mathrm{CI}-0.03$ to 0.15 ) while in the hypercapnic patients the PI tended to rise (baseline $P I=1.03(0.17), P I$ after protein load $=$ $1.12(0.21)$, mean difference $=-0.09,95 \%$ CI 0.18 to $0.007, p=0.06$ ).

Conclusions - Renal haemodynamics were unchanged after a protein load in patients with severe COPD, suggesting that they had no renal functional reserve. This may be a factor in the development of oedema frequently seen in patients with severe COPD, particularly in hypercapnic patients.

(Thorax 1997;52:411-415)
\end{abstract}

Keywords: chronic obstructive pulmonary disease, renal blood flow, Doppler ultrasound, renal functional reserve.
Oedema is a common complication of chronic obstructive pulmonary disease (COPD). The presence of oedema is a poor prognostic sign, with $60 \%$ of such patients being dead within five years. ${ }^{1}$ The pathogenesis of oedema in COPD remains unclear. It was originally hypothesised that these patients had right ventricular failure secondary to pulmonary hypertension as a consequence of hypoxia induced pulmonary vasoconstriction. However, in the absence of primary cardiac pathology, most patients with COPD have normal or even increased cardiac output. ${ }^{1}$ It now seems likely that the development of oedema in COPD may, at least in part, be due to non-cardiac factors such as changes in renal haemodynamics or hormonal imbalances. ${ }^{2-4}$

During acute oedematous exacerbations of COPD renal blood flow is reduced, especially in the presence of hypercapnia, ${ }^{235}$ but increases when the clinical status improves. ${ }^{35}$ In respiratory diseases generally, oedema is unusual in the absence of hypercapnia. ${ }^{4}$ Reduced renal blood flow activates the renin/angiotensin activating system, stimulating aldosterone release, with subsequent sodium and water retention. ${ }^{4}$

Most patients with COPD, even those with oedema, have normal serum creatinine levels with a normal or only slightly reduced glomerular filtration rate. $^{26}$ We therefore investigated whether a more sensitive index of renal function would show evidence of renal impairment in patients with COPD. The increase in glomerular filtration rate and renal blood flow in response to a protein load is known as the renal functional reserve which is often impaired early in the course of renal disease, frequently before there is a fall in glomerular filtration rate. Reduced renal functional reserve may thus be an early index of renovascular impairment. We hypothesised that, even if resting haemodynamics are normal in patients with moderate to severe stable COPD, the renal functional reserve may be reduced compared with controls, particularly in hypercapnic patients. We investigated this hypothesis using duplex ultrasonography which is a rapid, reproducible, and non-invasive method of studying renal haemodynamics.

\section{Methods}

Sixteen patients with severe stable COPD were recruited consecutively from our outpatient clinic. The principal inclusion criterion was a 
forced expiratory volume in one second $\left(\mathrm{FEV}_{1}\right)$ of $<1.21$. Ten of the 16 patients had a history of oedema, although only one had current oedema. They fulfilled the American Thoracic Society criteria for COPD, and had had no acute exacerbation or change in medication for at least six weeks. They were all current or previous cigarette smokers. Each patient gave informed consent and the study was approved by the hospital ethics committee. Patients with known diabetes mellitus, hypertension, liver or renal impairment, or current left ventricular failure were excluded from the study. Renal impairment was defined as serum creatinine levels of $>120 \mathrm{mmol} / 1$ and/or the presence of proteinuria. Current diuretic use was not an exclusion factor. Five normal controls were also recruited from the retired hospital staff members' association. The controls were nonsmokers with no chronic illness and on no medication; they also gave informed consent.

\section{ULTRASONOGRAPHY}

Doppler ultrasound examinations were performed using an Acuson 128 real time ultrasound scanner with colour flow and pulsed scanning facility. The patients fasted overnight and omitted their morning medication, including all inhaled therapy. With the patient in the seated position the right kidney was scanned in the longitudinal plane via the translumbar route with a $2 \mathrm{MHz}$ probe. ${ }^{78} \mathrm{~A}$ renal interlobar artery was identified both from its anatomical position and typical sonogram showing the characteristic high diastolic blood velocity. The angle of the ultrasound beam was adjusted until the maximum Doppler frequency shift was obtained. Good quality Doppler signals were obtained in all subjects. Doppler indices, including the pulsatility index (PI), were calculated using the integrated computer software. The PI is obtained by calculating the difference between the peak systolic frequency shift of the Doppler spectrum (A) and the end diastolic frequency shift (B), which is then divided by the mean frequency shift (mean) such that $\mathrm{PI}=$ $\mathrm{A}-\mathrm{B} /$ mean $^{9}{ }^{9} \mathrm{PI}$ is an index of distal resistance to flow in the vascular bed; the lower the PI, the less the resistance to flow and therefore the greater the rate of flow. It is independent of the vessel diameter and the angle between the Doppler beam and the vessel axis. There was little variation in the PI with each arterial pulsation, and the mean of a minimum of three PI measurements from the same interlobar artery was calculated.
MEASUREMENT PRECISION

The same experienced operator made all the renal blood flow measurements. The intraobserver variability of the non-invasive measurement of renal blood flow was determined by the same operator who took paired measurements of renal blood flow in 15 adults which were then compared using a Bland and Altman plot. ${ }^{10}$ The mean of the difference between the two measurements of PI was only 0.001 with a standard deviation of 0.026 . The $95 \%$ confidence limits for precision - that is, \pm 2 SD of the mean difference - were thus \pm 0.052 . The mean within subject coefficient of variation for PI was $2.05 \%$.

\section{PROTEIN LOAD}

Fasting baseline renal blood flow was measured. The patients then ate a $250 \mathrm{~g}$ steak containing approximately $70 \mathrm{~g}$ protein, without added salt, over a maximum of 30 minutes. They also drank $500 \mathrm{ml}$ water. The PI was recalculated $30,75,120$, and 165 minutes after eating the steak and the minimum PI obtained (or maximum value if the PI rose). The maximum change in PI from baseline was calculated in each subject which represented the renal functional reserve.

\section{DATA ANALYSIS}

The baseline PI value of each subject before the steak meal acted as the control value. As the data were normally distributed the Student's $t$ test for paired data was used to calculate the significance of differences between the baseline and maximum PI. Numerical variables were compared between the control subjects and patients with COPD by the Student's $t$ test for unpaired data. Variables were also compared by least squares regression analysis. The results are given as mean (SD) and a p level less than 0.05 was considered significant.

\section{Results}

Sixteen patients with COPD were studied, of whom 10 were men. Their pulmonary function and blood gas data are given in table 1 . Ten of the COPD group were hypercapnic $\left(\mathrm{PaCO}_{2}\right.$ $\geq 6.0 \mathrm{kPa})$ with a mean $\mathrm{PaCO}_{2}$ of $6.7(0.52)$ $\mathrm{kPa}$. Only one patient with COPD had oedema at the time of the study, although 10 patients had a history of previous oedema and were on long term diuretic therapy, four of whom were

Table 1 Mean (SD) age, pulmonary function, and blood gas data for patients with chronic obstructive pulmonary disease (COPD) and normal controls

\begin{tabular}{lcccc}
\hline & $\begin{array}{l}\text { COPD } \\
(n=16)\end{array}$ & $\begin{array}{l}\text { Controls } \\
(n=5)\end{array}$ & $\begin{array}{l}\text { Hypercapnic COPD } \\
(n=10)\end{array}$ & $\begin{array}{l}\text { Normocapnic COPD } \\
(n=6)\end{array}$ \\
\hline Age (years) & $65.9(5.7)$ & $63.0(6.04)$ & $64.1(6.04)$ & $68.8(3.87)$ \\
FEV (1) & $0.7(0.23)$ & $2.81(0.42)^{* * *}$ & $0.73(0.19)$ & $0.65(0.29)$ \\
FVC (1) & $1.95(0.67)$ & $35.62(0.56)^{* * *}$ & $1.84(0.61)$ & $2.13(0.78)$ \\
FEV $/$ FVC (\%) & $37.1(9.98)$ & $75.2(7.3)^{* * *}$ & $41.6(9.54)$ & $30.3(6.53)^{*}$ \\
$\mathrm{KCO}_{\text {(\% predicted) }}$ & $46.8(26.2)$ & $86.0(5.7)^{* * *}$ & $59.8(22.4)$ & $23.4(12.4)^{* *}$ \\
$\mathrm{PaO}_{2}(\mathrm{kPa})$ & $8.1(1.04)$ & & $7.9(1.1)$ & $8.8(0.91)$ \\
$\mathrm{PaCO}_{2}$ (kPa) & $6.3(0.69)$ & & $6.7(0.52)$ & $5.6(0.35)^{* * *}$
\end{tabular}

$\mathrm{FEV}_{1}=$ forced expiratory volume in one second $\mathrm{FVC}=$ forced vital capacity; $\mathrm{KcO}=$ lung diffusion capacity corrected for alveolar volume; $\mathrm{PaO}_{2}, \mathrm{PaCO}_{2}=$ arterial oxygen and carbon dioxide tensions.

The COPD group were subdivided into hypercapnic $\left(\mathrm{PaCO}_{2} \geq 6.0 \mathrm{kPa}\right)$ and normocapnic $\left(\mathrm{PaCO}_{2}<6.0 \mathrm{kPa}\right)$ subjects. ${ }_{\mathrm{p}}<0.05, * * \mathrm{p}<0.01, * * * \mathrm{p}<0.001$ COPD group versus controls and hypercapnic COPD versus normocapnic COPD patients. 


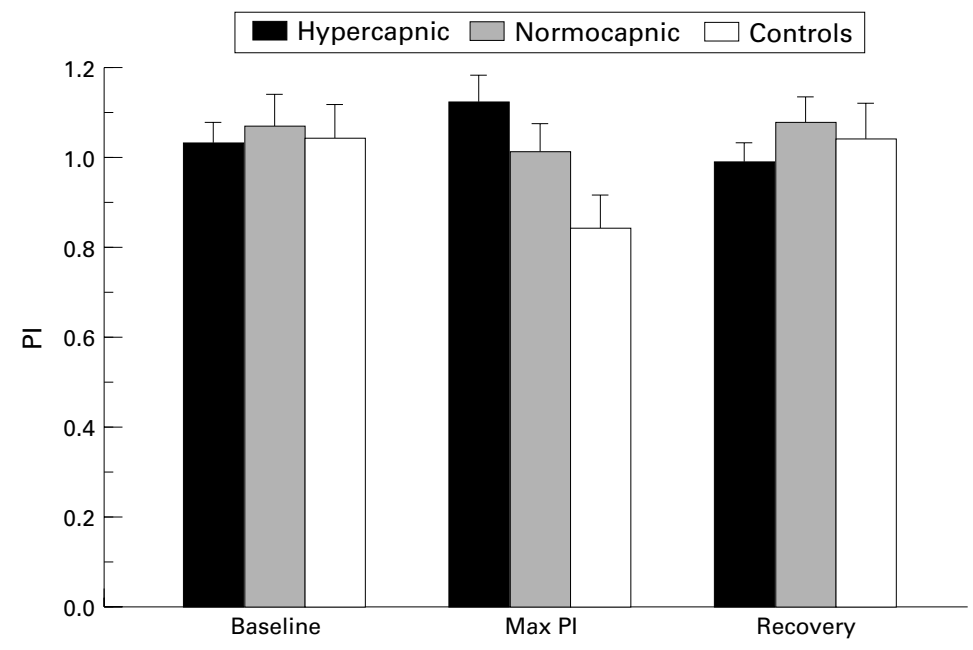

Figure 1 Mean changes in pulsatility index (PI) after a protein meal in hypercapnic and normocapnic patients with COPD and in control subjects. Maximum PI is the point of maximum change in PI from baseline after the protein load. Recovery PI is the PI at 165 minutes after the protein load.

also on angiotensin converting enzyme (ACE) inhibitors. Five patients with COPD (three of whom were hypercapnic) were on long term home oxygen therapy and nine patients had home nebulisers. Of the five normal controls, three were men.

RENAL RESPONSE TO PROTEIN LOAD

The baseline (fasting) PI was similar in the control group and in the patients with COPD. The PI fell in all the control subjects after the protein meal by a mean of $19.2 \%$ from an initial value of $1.04(0.19)$ to $0.84(0.17)$ (mean difference $0.20,95 \%$ confidence interval of difference (CI) 0.14 to $0.27, \mathrm{p}<0.001$; fig $1)$. In the COPD group there was a variable response to the protein load with the group as a whole showing no significant change in PI (baseline $1.04(0.16)$ rising to $1.08(0.19)$, mean difference $-0.04,95 \% \mathrm{CI}-0.11$ to 0.04 , $\mathrm{p}=\mathrm{NS}$ ). This suggests that the patients with COPD had no renal functional reserve. The change in PI in response to the protein meal was significantly greater in the control group than in the patients with COPD ( $\mathrm{p}<0.001)$.

The COPD group was subdivided into 10 hypercapnic and six normocapnic subjects (table 1). In the normocapnic subjects the PI fell by $0.06(5.6) \%$ after the steak ingestion from $1.07(0.15)$ to $1.01(0.16)$ (mean difference $0.06,95 \%$ CI -0.03 to $0.15, p=N S$ ), while in the hypercapnic subjects the PI rose by $0.09(8.7) \%$ from $1.03(0.17)$ to $1.12(0.21)$ (mean difference $-0.09,95 \%$ CI 0.18 to 0.007) which approached statistical significance $(p=0.06)$. The change in PI was significantly greater in the hypercapnic than in the normocapnic patients $(p<0.05)$. Of the 10 hypercapnic patients the PI rose after the protein meal in eight cases, was unchanged in one, and fell in only one subject. The maximum change in PI in all the controls was at 75 minutes after steak ingestion, while in the COPD group the peak change occurred later with only four of the 15 patients reaching their maximum change in PI by 75 minutes $(p<0.01$, Fisher's exact test).

There was no correlation between the $\mathrm{PaO}_{2}$ and the maximum change in PI in the patients with COPD $(r=0.06, \mathrm{p}=\mathrm{NS})$ but a stronger relationship was found between the $\mathrm{PaCO}_{2}$ and the maximum change in PI $(r=-0.34, \mathrm{p}$ $<0.05)$. There was no correlation between the baseline PI and the $\mathrm{PaO}_{2}$ or $\mathrm{PaCO}_{2}$, nor was there any correlation between the baseline or change in PI and age.

\section{Discussion}

Baseline renal haemodynamics were found to be similar in patients with severe stable COPD and a control group. The control subjects had a fall in distal renovascular resistance after the protein load, implying less vasoconstriction and greater renal blood flow. This suggests that they had an intact renal functional reserve. However, there was no change in renal resistance following the protein load in the COPD group as a whole. Furthermore, in the hypercapnic COPD patients the distal renal resistance increased rather than decreased in response to protein loading. This suggests that patients with severe COPD, particularly those who are hypercapnic, have no renal functional reserve, indicative of abnormal renovascular responses.

It is known that the normal kidney has the ability to increase the glomerular filtration rate and renal blood flow in response to certain stimuli such as an oral protein load, amino acid infusion and dopamine infusion. ${ }^{11-14}$ This phenomenon is known as the renal functional reserve and is defined as the difference between the baseline glomerular filtration rate and/or renal blood flow and the maximum value after a protein load. ${ }^{12}{ }^{15}$ A $250 \mathrm{~g}$ steak has been successfully used as a protein load in previous studies. ${ }^{1216}$ Renal functional reserve is an index of the capacity of the kidney to increase its function by vasodilatation of glomerular arterioles and recruitment of dormant nephrons. Glomerular hyperfiltration secondary to a high protein diet is known to accelerate the progression of renal disease. ${ }^{17}$ The mechanisms that cause an increase in both glomerular filtration rate and renal blood flow following protein loading in humans are at present unknown, but the hormones implicated include glucagon, glomerulopressin, prostaglandins, and angiotensin-2. ${ }^{18}$ Changes in the tubular handling of sodium may also play a part.

There is no constant value for renal functional reserve as it has been shown to vary between $10 \%$ and $70 \%$ of the baseline glomerular filtration rate. ${ }^{19}$ Likewise, there is no constant relationship between the glomerular filtration rate and changes in renal functional reserve; the renal functional reserve may be decreased before any change occurs in the glomerular filtration rate, or it may fall linearly or variably with the glomerular filtration rate. ${ }^{20} \mathrm{As}$ progressive renal impairment occurs the renal functional reserve reaches a zero value at some decreased level of baseline glomerular filtration rate. $^{20}$ The impairment or absence of renal 
functional reserve may therefore be an early indication of impaired renal function. To our knowledge there are no previous studies of renal functional reserve in patients with COPD.

Pulsed Doppler ultrasonography is a relatively new technique which has made rapid, repeated, direct, non-invasive measurement of renal blood flow possible. The detection of blood flow in the renal interlobar artery can be difficult in the aged obese patient and in those with renal impairment, but the detection rate is higher using the translumbar route. ${ }^{21}$ Each recording takes only 2-3 minutes after identification of the interlobar artery and the results are instantly available. The equipment is moderately expensive and experience is required to obtain optimal results. However, in most of the subjects we had no difficulty in obtaining sonograms of satisfactory quality. Our intraobserver precision studies showed linear agreement between repeated measurements of PI with $95 \%$ confidence limits for accuracy of 0.052 and a coefficient of variation of $2.05 \%$. As all the significant changes in PI were within the precision of our method, one can be $95 \%$ confident that the significant changes in PI were real.

Our methodology allowed us to measure changes in renal resistance only, using the PI, rather than giving us an absolute measurement of renal blood flow. To measure renal blood flow directly requires calculation of both the vessel diameter and the angle between the Doppler beam and the direction of flow. The use of Doppler ultrasonography to assess renal haemodynamics has been validated against perivascular Doppler cuffs in dogs. ${ }^{11}$ In humans it has been validated against PAH clearance. ${ }^{112223}$ It has also been used to detect rapid changes in blood flow in the renal interlobar artery in response to dopamine infusion ${ }^{823}$ and oxygen administration in patients with COPD. ${ }^{723}$ Unlike PAH clearance, Doppler ultrasonography is not dependent on normal renal tubular function and it allows direct instantaneous assessment of renal haemodynamics. The PI of the interlobar artery has been used previously in studies of renal haemodynamics in patients with $\mathrm{COPD}^{7}$ and in response to dopamine infusion. ${ }^{8}$ The PI is an index of resistance to flow in vascular beds distal to the point of sampling, thus it is an indirect index of the degree of vasoconstriction. The fall in PI in our control subjects suggests that they had renal vasodilatation following the protein meal which failed to occur in the normocapnic COPD patients, while the hypercapnic COPD patients had increased vasoconstriction after protein loading.

The mechanisms causing the oedema of COPD are not fully known. Early studies proposed that the oedema was due simply to right ventricular failure secondary to hypoxaemic pulmonary hypertension. ${ }^{4}$ However, in hypoxaemic interstitial lung disease oedema rarely develops until the terminal stages are reached. Furthermore, many COPD patients with oedema have been found to have no evidence of right ventricular hypertrophy at necropsy, ${ }^{4}$ and cardiac output is usually normal or only slightly reduced. ${ }^{424}$ It is now thought that oedema formation in patients with COPD is multifactorial in origin. A review of 150 cases of COPD found that most of the patients with oedema had hypercapnia. ${ }^{25}$ The authors hypothesised that the hypoventilation in these patients leads to respiratory acidosis which increases renal tubular exchange of hydrogen ion for sodium and reabsorption of bicarbonate, leading eventually to fluid retention. Hypercapnia appears to reduce renal blood flow, ${ }^{2326}$ while studies on the effects of hypoxaemia have shown variable results. ${ }^{723} 26$

Reduced renal blood flow in the presence of normal glomerular filtration rate reduces the filtration fraction, leading to sodium and water retention. ${ }^{27}$ Reduced renal blood flow also activates the renin-angiotensin activating system, increasing plasma aldosterone levels with further sodium and water retention and the development of oedema. ${ }^{24}$ The variable increase in these hormones frequently found in patients with COPD during acute exacerbations ${ }^{23}$ appears to be secondary to the haemodynamic changes induced by hypercapnia rather than a primary effect of hypercapnia per se. ${ }^{324}$ Levels of antidiuretic hormone are also often inappropriately high for the plasma osmolarity in COPD patients with oedema. ${ }^{2}$

Hypercapnia can reduce renal blood flow by several mechanisms. Firstly, it may cause direct renal vasoconstriction. ${ }^{26}$ Secondly, hypercapnia causes peripheral vasodilatation which inactivates the baroreceptors with subsequent noradrenaline release which causes reduced renal blood flow. ${ }^{34}$ Hypercapnia also increases noradrenaline levels by direct central sympathetic stimulation. ${ }^{3}$ Cardiac output is not reduced by hypercapnia ${ }^{324}$ so the reduced renal blood flow caused by hypercapnia is more likely to be due to renal vasoconstriction rather than secondary to a fall in cardiac output. Howes et $a l$, in an ultrasound study of patients with stable COPD, found that hypercapnic patients had reduced renal blood flow while the renal blood flow was similar in normocapnic patients and control subjects. ${ }^{23}$ However, in our study we found no significant differences in baseline renal haemodynamics between the patients with COPD and the controls, possibly because our COPD patients had less severe respiratory failure with a mean $\mathrm{PaO}_{2}$ of $8.1(1.04) \mathrm{kPa}$ and $\mathrm{PaCO}_{2}$ of $6.3(0.69) \mathrm{kPa}$, and only two patients had a $\mathrm{PaCO}_{2}$ of $\geqslant 7.0 \mathrm{kPa}$.

The number of patients with COPD in our study was small for subgroup analysis with only 10 hypercapnic and six normocapnic patients. However, the decision to do subgroup analysis was part of the study design, as one of our hypotheses was that there may be greater impairment of the handling of a protein load in hypercapnic rather than in normocapnic patients. We feel that the results are of interest as they help to show the abnormal renal haemodynamics that occur in patients with hypercapnia, and the findings are consistent with other studies. ${ }^{23}$ Howes et al, also using Doppler ultrasound, have shown that renal blood flow increased acutely in response to oxygen and dopamine infusion in normal controls and in 
patients with normocapnic hypoxaemic COPD. ${ }^{23}$ However, there was no change in the renal blood flow in hypercapnic COPD patients. This is consistent with our findings, as the increase in renal blood flow in response to dopamine infusion could be regarded as a measurement of renal functional reserve, although this issue was not specifically addressed in that study. Reduced renal functional reserve suggests an inability to increase renal blood flow and a lack of renal reserve, and such patients may be more prone to reduced renal blood flow during acute exacerbations with the development of frank oedema. Furthermore, reduction in renal functional reserve implies a chronic hyperfiltration state which in itself can accelerate renal damage. ${ }^{17}$

Howes et al found that the rise in renal blood flow in response to oxygen and dopamine in their normocapnic patients was not accompanied by any increase in cardiac output. ${ }^{23}$ Conversely, in the present study it is unlikely that the absence of renal functional reserve was due to reduced baseline cardiac output as cardiac output is usually normal in patients with COPD and the patients were selected on the basis of absent cardiac disease. The hypercapnic patients in our study had an increase rather than the expected decrease in renal vascular resistance following a protein load. Avasthi et al have also investigated postprandial renal blood flow using Doppler methodology ${ }^{11}$ and found that renal vascular resistance decreased in normal subjects after a protein-rich meal, suggesting increased renal blood flow, while cardiac output increased to an even greater extent. They hypothesised that the rise in cardiac output was necessary to provide increases in postprandial superior mesenteric artery blood flow. It is possible that in our hypercapnic patients there was an insufficient increase in cardiac output to increase blood supply to both the superior mesenteric artery and renal arteries leading to a diversion of blood flow away from the kidneys.

Four of our patients, all of whom were hypercapnic, had been taking ACE inhibitors prior to the study. They all omitted their medication on the day of the study. To our knowledge, there are no studies on the effects of ACE inhibitors on renal functional reserve. Following chronic use in patients with COPD, ACE inhibitors cause a small but significant increase in effective renal plasma flow while having no effect on the glomerular filtration rate. ${ }^{28}$ This suggests that ACE inhibitors are unlikely to prevent the usually expected increase in renal blood flow in response to protein loading. Furthermore, when the patients who were on ACE inhibitors were removed from the data analysis the results were not affected.

In summary, in this non-invasive study we found that renal resistance decreased after a protein meal in a control group of subjects. This suggests that they had increased renal blood flow and intact renal functional reserve. However, renal resistance was unchanged in patients with severe stable normocapnic COPD but was increased in patients with hypercapnic
COPD. This absence of renal functional reserve suggests the presence of impaired renal haemodynamics in patients with COPD which may play a part in the frequent development of oedema in such patients.

1 Hodgkin JE. Prognosis in chronic obstructive pulmonary disease. Clin Chest Med 1990;11:555-69.

2 Farber MO, Roberts LR, Weinberger MH, Robertson GL, Manfredi F. Abnormalities of sodium and $\mathrm{H}_{2} \mathrm{O}$ handling in chronic obstructive lung disease. Arch Intern Med 1982; 142:1326-30

3 Anand IS, Chandrashekhar Y, Ferrari R, Sarma S, Guleria R, Tindal SK, et al. Pathogenesis of congestive state in chronic obstructive pulmonary disease. Circulation 1992; 86:12-21.

4 MacNee W. Pathophysiology of cor pulmonale in chronic obstructive pulmonary disease. Am f Respir Crit Care Med 1994;150:833-52; 1158-68.

5 Platts MM, Hammond JDS, Stuart-Harris CH. A study of cor pulmonale in patients with chronic bronchitis. $Q \mathcal{F}$ Med 1960;29:559-74

6 Fishman AP, Maxwell MH, Crowder CH, Morales P. Kidney function in cor pulmonale: particular consideration of changes in renal hemodynamics and sodium excretion during variation in level of oxygenation. Circulation 1951 3:703-21.

7 Baudouin SV, Bott J, Ward A, Deane C, Moxham J. Short term effect of oxygen on renal haemodynamics in patients with hypoxaemic chronic obstructive airways disease. Thorax 1992;47:550-4.

8 Stevens PE, Gwyther SJ, Bouletbee JE, Bolsin S, Hanson ME, Kox W. Practical use of duplex Doppler analysis of the renal vasculature in critically ill patients. Lancet 1989; i: $240-2$.

9 Evans DH, McDicken WM, Skidmore R, Woodcock JP. Doppler ultrasound. Physics, instrumentation and clinical appoppler ultrasound. Physics, instrumentation and

10 Bland JM, Altman DG. Statistical methods for assessing agreement between two methods of clinical measurement. Lancet 1986;i:307-10.

11 Avasthi PS, Greene ER, Voyles WF. Noninvasive Doppler assessment of human postprandial renal blood flow and cardiac output. Am f Physiol 1987;252:F1167-74

12 Bosch JP, Lew S, Glabman S, Lauer A. Renal hemodynamic changes in humans. Am f Med 1986;81:809-15.

13 Graf H, Stummvoll HK, Luger A, Prager R. Effect of aminoacid infusion on glomerular filtration rate. $N$ Engl f Med 1983;308:159-60.

14 Ter Wee PM, Smit AJ, Rosman JB, Sluiter WJ, Donker AJM. Effect of intravenous infusion of low-dose dopamine on renal function in normal individuals and in patients with renal disease. Am F Nephrol 1986;6:42-6.

15 Memoli B, Libetta C, Sabbatini M, Conte G, Russo D, Giani U, et al. Renal functional reserve: its significance in normal and salt depletion conditions. Kidney Int 1991;40 $1134-40$.

16 Hostetter TH. Human renal response to a meat meal. $A m$ F Physiol 1986;250:F613-8.

17 Brenner BM, Meyer TW, Hostetter TH. Dietary protein intake and the progressive nature of kidney disease: the role of hemodynamically mediated glomerular injury in the pathogenesis of progressive glomerular sclerosis in ageing, renal ablation, and intrinsic renal disease. $N$ Engl fMed 1982;307:652-9.

18 Woods LL. Mechanisms of renal hemodynamic regulation in response to protein feeding. Kidney Int 1993; 44:659-75.

19 Ter Wee PM, Geerlings W, Rosman JB, Sluiter WJ, van der Geest S, Donker AJM. Testing renal reserve filtration capacity with an amino acid solution. Nephron 1985;41: capacity

20 Amiel C, Blanchet F, Freidlander G, Nitenberg A. Renal functional reserve. Nephrol Dial Transplant 1990;5:763-70

21 Handa N, Fukunaga R, Etani H, Yoneda S, Kimura K, Kamada T. Efficacy of echo-Doppler examination of the evaluation of renovascular disease. Ultrasound Med. Bio 1988;1:1-5

22 Yura T, Takamitsu Y, Yuasu S, Miki S, Takahashi N, Bandai $\mathrm{H}$, et al. Total and split renal function assessed by ultrasound Doppler techniques. Nephron 1991;58:37-41.

23 Howes TQ, Deane CR, Levin GE, Baudouin SV, Moxham . The effects of oxygen and dopamine on renal and aortic blood flow in chronic obstructive pulmonary disease with hypoxemia and hypercapnia. Am 7 Respir Crit Care Med 1995;151:378-83.

24 Chabot F, Mertes PM, Delorme N, Schrijen FV, Saunier CG, Polu JM. Effect of acute hypercapnia on alpha atrial natriuretic peptide, renin, angiotensin II, aldosterone, and vasopressin plasma levels in patients with COPD. Chest 1995;107:780-6.

25 Campbell EJM, Short DS. The cause of oedema in 'cor pulmonale'. Lancet 1960; i:1184-6.

26 Kilburn KH, Dowell AR. Renal function in respiratory failure. Effects of hypoxia, hyperoxia and hypercapnia. Arch Intern Med 1982;127:754-62.

27 Hall JE. Regulation of renal hemodynamics. In: Guyton AC, Hall JE, eds. Cardiovascular physiology IV. Vol 26 International review of physiology. Baltimore: University Park Press, 1982:243-321.

28 Oliver RM, Peacock AJ, Fleming J, Waller DG. Rena and pulmonary effects of angiotensin converting enzyme inhibition in chronic hypoxic lung disease. Thorax 1989 44:513-5. 